\title{
Un modèle-outil pour représenter le savoir technopédagogique disciplinaire des enseignants
}

\section{Sylviane Bachy}

\section{(2) OpenEdition}

\section{Journals}

Édition électronique

URL : http://journals.openedition.org/ripes/821

DOI : 10.4000/ripes.821

ISSN : 2076-8427

Éditeur

Association internationale de pédagogie universitaire

Référence électronique

Sylviane Bachy, «Un modèle-outil pour représenter le savoir technopédagogique disciplinaire des enseignants », Revue internationale de pédagogie de l'enseignement supérieur [En ligne], 30(2) | 2014, mis en ligne le 10 mai 2014, consulté le 10 décembre 2020. URL : http://journals.openedition.org/ripes/ 821 ; DOl : https://doi.org/10.4000/ripes.821

Ce document a été généré automatiquement le 10 décembre 2020.

Article L.111-1 du Code de la propriété intellectuelle. 


\title{
Un modèle-outil pour représenter le savoir technopédagogique disciplinaire des enseignants
}

\author{
Sylviane Bachy
}

\section{Introduction}

1 Cette étude a pour objectif de proposer un modèle théorique intégrateur servant à comprendre les différentes influences qui déterminent les pratiques pédagogiques des enseignants. Sur la base de plusieurs modèles existants, nous proposons un outil permettant de dresser des profils d'enseignants en ce qui concerne l'influence de leur culture disciplinaire, de leur épistémologie personnelle, de leurs connaissances pédagogiques et de leurs connaissances technologiques. Ceci est mené afin de guider davantage le travail des formateurs d'enseignement (conseillers pédagogiques, enseignants, maîtres de formation pratique) pour mieux comprendre pourquoi certaines tâches sont utilisées plutôt que d'autres par un enseignant. Il s'agit de répondre à la question suivante : pourquoi (sous quelles influences) l'enseignant fait-il tel ou tel choix dans ses pratiques pédagogiques? Ainsi, le conseiller ou le formateur peut davantage cibler ses actions en fonction des références ou des représentations que les enseignants ont sur leurs pratiques pédagogiques.

2 Pour aborder cette thématique, notre posture épistémologique se situe au croisement du champ de recherche didactique et du champ de recherche pédagogique. Toutefois, cette communication se limitera à définir un modèle-outil permettant de guider le travail pédagogique des formateurs d'enseignants à l'université. Jusqu'il y a peu, les recherches en sciences de l'éducation portaient essentiellement sur les références pédagogiques en fonction des contenus à enseigner (Shulman, 1986, 1987, 1998, 2004, 2007) ou sur les influences des croyances des enseignants à propos de l'acte d'enseigner (Vause, 2009). Au moment où les développements technologiques vont bon train, il 
nous semblait important de requestionner ces différentes influences et d'analyser comment les connaissances technologiques des enseignants s'intègrent à celles-ci.

C'est la raison pour laquelle, la première partie de cet article présente tout d'abord deux outils conceptuels. Le premier modèle Pedagogical Content Knowledge de Shulman (1986) fait partie des travaux les plus importants, selon nous, pour aborder l'impact des disciplines (dimension disciplinaire) et des connaissances pédagogiques (dimension pédagogique) sur les pratiques d'enseignement. Ce modèle a fait l'objet de plusieurs révisions et de plusieurs adaptations. L'une d'entre elles a retenu toute notre attention. Il s'agit du deuxième modèle sur lequel nous nous appuyons: le savoir pédagogique disciplinaire de Berthiaume (2007). L'auteur propose un outil spécifique pour les enseignants de l'université où il ajoute la dimension de l'épistémologie personnelle aux deux premières dimensions présentes dans le modèle de Shulman.

Dans la deuxième partie, nous explorons les pistes qui intègrent les technologies à la réflexion. Ainsi, le modèle Technological and Pedagogical Content Knowledge (Mishra \& Koehler, 2006) compile les dimensions disciplinaires, pédagogiques et technologiques sans tenir compte des croyances des enseignants par rapport à l'apprentissage ou à la construction des savoirs. Sur la base de ces différents apports théoriques, nous proposons un modèle-outil intégrateur: le savoir technopédagogique disciplinaire. Après l'avoir décrit, les parties suivantes de l'article présentent les analyses de validation qui ont été menées auprès d'enseignants de l'enseignement supérieur universitaire et non-universitaire. Enfin, nous discuterons des liens entre les différentes dimensions et des perspectives d'usage de ce modèle-outil pour le conseil pédagogique ou pour des recherches à venir.

\section{Pédagogie et Contenu}

Shulman (1986) a mis en évidence les processus de transformation des savoirs qui concernent le contenu. Il a introduit le concept de Pedagogical Content Knowledge (PCK).

\subsection{Les connaissances pédagogiques du contenu - PCK}

6 Le PCK se traduit en français par les connaissances pédagogiques du contenu (Shulman, 2007) ou par savoir didactique, " puisque qu'on peut le définir comme la connaissance que l'on a d'un savoir à des fins d'enseignement » (Sensevy, 2009, p.53).

7 Il s'agit d'une forme de savoir pratique utilisée par les enseignants pour guider leurs actions. Pour Shulman (1986), ce savoir comprend:

1. Une compréhension de la façon de structurer et de présenter le sujet à apprendre ;

2. Une compréhension des conceptions communes ou spontanées, des idées fausses et les difficultés que rencontrent les apprenants lors de l'apprentissage d'un sujet particulier ;

3. Une connaissance des stratégies d'enseignement qui sont efficaces pour répondre aux besoins d'apprentissage des étudiants dans des circonstances particulières en classe.

8 L'enseignement dépend de l'organisation de systèmes des connaissances: celles qui touchent à l'apprentissage et à l'enseignement et celles qui touchent au contenu. Historiquement, on avait tendance à parfois différencier ces deux dimensions. Les conséquences pratiques de l'isolement de ces deux dimensions ont donné des profils d'enseignant centrés soit sur le contenu (C), soit sur la pédagogie (P). 
9 Les connaissances du contenu comprennent les connaissances qui portent sur la matière qui doit être apprise ou enseignée (Schwab, 1964). Les enseignants doivent avoir la connaissance des sujets qu'ils enseignent incluant les faits majeurs, les concepts, les théories et les procédures dans leur champ disciplinaire. Nous disposons déjà de nombreuses manières pour représenter la connaissance du contenu; par exemple, la taxonomie de Gagné (1985) propose de structurer les différents contenus à enseigner en faits, concepts, procédures et stratégies.

Les connaissances pédagogiques comprennent les connaissances sur les méthodes d'enseignement et d'apprentissage, la manière de gérer la classe, les évaluations, etc. L'enseignant qui a de bonnes connaissances pédagogiques comprend comment les étudiants construisent leurs connaissances, acquièrent des compétences et développent de bonnes habitudes au regard de l'apprentissage (Mishra \& Koehler, 2006).

11 Les travaux de Shulman (1986) ont visé à créer une intersection entre le contenu et le pédagogique. Selon Shulman (1987), avoir la connaissance du sujet traité et des stratégies pédagogiques générales n'est pas suffisant pour qualifier de bons enseignants. Les connaissances pédagogiques du contenu (PCK) rendent mieux compte de la manière dont certains contenus devraient être enseignés. "La connaissance pédagogique du contenu inclut aussi une compréhension de ce qui rend facile ou difficile l'apprentissage de sujets spécifiques: les conceptions ou préconceptions que les élèves d'âges divers et de différentes cultures portent avec eux dans l'apprentissage des domaines et leçons les plus fréquemment enseignés » (Shulman, 2007, p.105). Selon Mishra et Koehler (2006), le cœur du PCK est la manière de transformer l'objet pour l'enseigner.

Figure 1. PCK - Adapté de Shulman (1986).

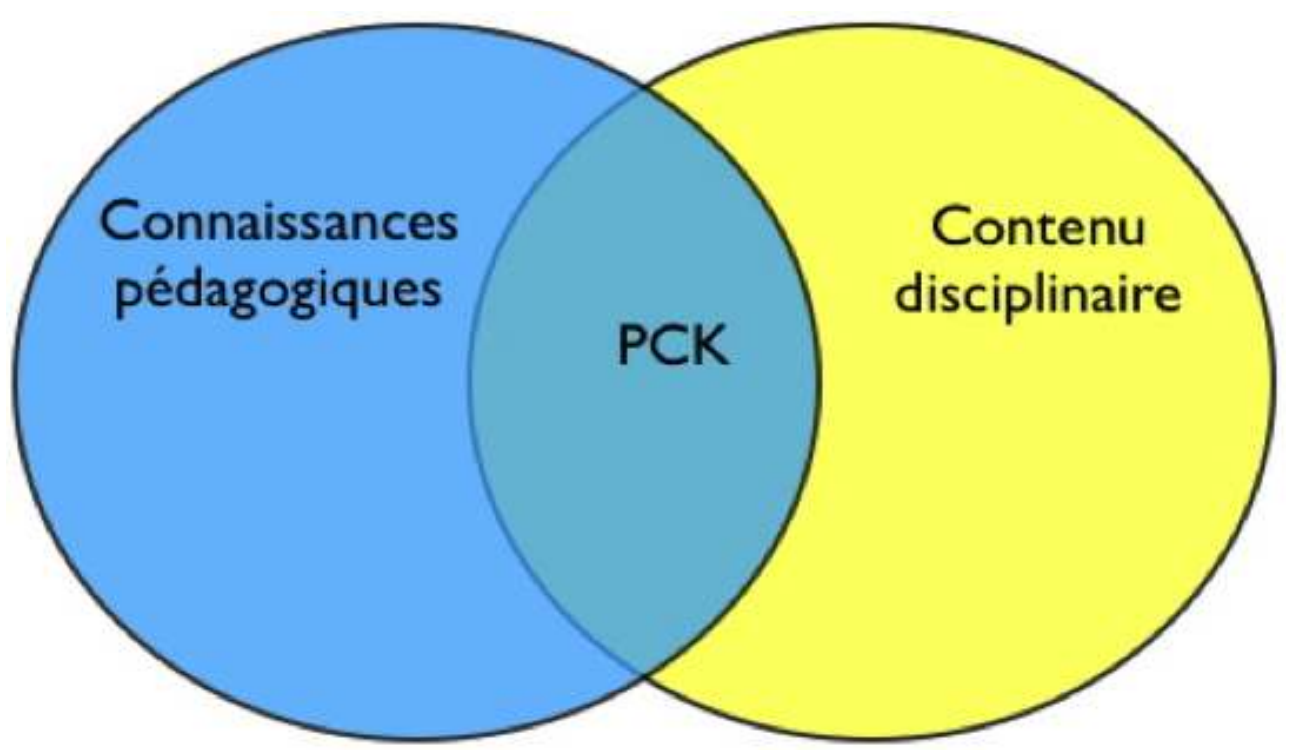

Notons que le PCK, tel que proposé par Shulman $(1986,1987)$, est particulièrement intéressant parce qu'il propose, pour la première fois, d'expliquer les pratiques des enseignants. Il combine le contenu et la pédagogie pour comprendre comment certaines thématiques sont représentées et adaptées pour qu'elles soient apprises. Il s'agit d'étendre les connaissances du savoir en connaissances pour enseigner le savoir. 
13 L'influence des recherches de Shulman dans le monde anglo-saxon et américain est très grande aujourd'hui. De nombreux travaux (Amade-Escot, 2001; Gess-Newsome \& Lederman, 1999 ; Segall, 2004) reposent encore sur son modèle. Pour Sensevy (2009), ces avancées anglo-saxonnes peuvent nous aider par leur pragmatisme. Elles étudient les pratiques telles qu'elles se font et permettent des coopérations avec les professeurs pour la définition de dispositifs spécifiques. Elles « intègrent comme sans y penser, au sein d'une même recherche, des considérations qu'on s'attendait plutôt, dans les études francophones, à voir dispersées tantôt dans les recherches de sciences de l'éducation tantôt dans des recherches de didactiques » (Sensevy, 2009, p.53).

La notion de PCK a été étendue et critiquée par la suite par de nombreuses études. Par exemple, les travaux de Cochran, King et De Ruiter (1993), van Driel, Verloop et Devos (1998), Segall (2004) ou encore Shulman lui-même (2007), qui a ajouté aux connaissances du contenu et aux connaissances pédagogiques :

- Le savoir sur le curriculum (issu des programmes);

- Le savoir concernant les apprenants ;

- Le savoir ayant trait aux contextes éducatifs ;

- Le savoir portant sur les finalités éducatives ;

- Le savoir propre de la profession.

15 De plus, le modèle de Shulman qui intègre contenu et pédagogie a surtout fait l'objet d'études dans les niveaux d'enseignement primaire et secondaire (Magnusson, Krajcik \& Borko, 1999). Pour le niveau universitaire, Lenze (1995) a transformé le PCK en Discipline-specific Pedagogical Knowledge (DPK) afin de mieux tenir compte des particularités de ce niveau d'enseignement.

\subsection{Les particularités de l'enseignement à l'université}

Dans une approche multicas à l'université, Lenze (1995) a examiné le PCK chez des enseignants en linguistique et chez des enseignants de la langue espagnole. Elle a préféré renommer le modèle de Shulman pour davantage l'accorder aux profils spécifiques des enseignants de l'université en proposant Discipline-specific Pedagogical Knowledge. Elle justifie cela par le fait que le contenu enseigné par des professeurs d'université correspond en fait aux disciplines académiques en tant que telles. Dès lors, contenu et discipline sont envisagés comme des termes équivalents.

17 Cette adaptation du modèle de Shulman (1986) pour le public universitaire nous renforce dans l'idée qu'il existe des spécificités disciplinaires qui influencent les connaissances pédagogiques des enseignants de ce niveau et que celles-ci ne peuvent pas être ignorées si l'on souhaite comprendre les pratiques d'enseignement en ligne. Cela dit, dans le contexte universitaire, d'autres études sont venues compléter ce premier principe pour tenir compte de la diversité des profils des enseignants.

18 L'hétérogénéité des pratiques et des profils professoraux est généralement expliquée par l'impact de nombreux facteurs tant personnels que contextuels et organisationnels. À ce propos, Bourgeois (1990), relève cette hétérogénéité comme un aspect très spécifique de l'université. Il est à ce point difficile de caractériser les différences que Guyot et Bonami (2000) préfèrent parler de fédération plutôt que de profession. Ce qui permet de tenir compte des différents profils de profession en fonction des disciplines, des départements, d'unités «chacun développant son propre système de représentation, sa méthode de travail et de gestion des tâches, ses paradigmes, sa 
culture spécifique, sa clientèle, sa symbolique, ses technologies et ses logiques d'action » (p.7).

19 Cette hétérogénéité amène Guyot et Bonami à considérer également que le travail professoral universitaire a un ancrage disciplinaire prononcé. À ce sujet, ils déterminent quatre approches interprétatives :

- L'approche épistémologique : selon Biglan (1973), il existe trois axes pour déterminer les mécanismes de structuration des sciences et les rapports entre les disciplines. Il différencie les sciences dures des sciences douces/molles, les sciences pures des sciences appliquées et les sciences du vivant des sciences de l'inanimé. Selon Guyot et Bonami (2000), les caractéristiques épistémologiques de la discipline pourraient induire chez les enseignants qui en relèvent une certaine conception spécifique du savoir et dès lors influencer leur conception sur les moyens pour le transmettre ;

- L'approche culturaliste : selon Kuhn (1962), d'un point de vue sociologique, il s'agit d'une communauté de chercheurs qui partagent un certain nombre de paradigmes, des façons de voir les choses. Ils partagent des croyances, des techniques et des principes qui déterminent la culture disciplinaire (Becher, 1981 ; Kolb, 1981). Ces cultures disciplinaires peuvent ellesmêmes être divisées en sous-cultures dans une même faculté universitaire, notamment en ce qui concerne les modes de vie, les orientations d'enseignement ou les valeurs éducatives. Ces sous-cultures ne sont pas totalement étrangères les unes par rapport aux autres. Elles partagent des éléments communs régis par le cadre institutionnel et national ;

- L'approche par la contingence : il s'agit d'analyser les structures organisationnelles en fonction des contraintes environnementales. Ainsi, on peut analyser les disciplines scientifiques et l'université en fonction de leurs relations avec l'environnement extérieur. L'université dépend, en effet, des ressources de la société et doit produire sa recherche en tenant compte de sa pertinence sociale, de son cout, de son utilité. Selon Guyot et Bonami (2000), cette approche pourrait aussi permettre de rendre compte de la dimension qualitative des divergences pour ce qui est de l'implication dans les tâches d'enseignement ;

- L'approche structuro-fonctionnaliste : Bourdieu (1984) a montré que deux principes de hiérarchisation antagonistes structurent le champ universitaire. Il s'agit, d'une part, du principe scientifique (qui repose sur des ressources intellectuelles) et, d'autre part, du principe social (qui repose sur des ressources sociales). En fonction des disciplines, il a montré qu'en France, un principe prend généralement plus d'importance que l'autre. Au regard de ces travaux, il semblerait aisé d'expliquer que les disciplines où prévaut le principe scientifique (comme c'est le cas pour les sciences) s'investiront dans leurs ressources intellectuelles au détriment de ressources plus pédagogiques. À l'inverse, Guyot et Bonami (2000) évoquent les acteurs de disciplines comme le droit où le principe social serait prédominant, et qui s'investiraient sans retenue dans les tâches d'enseignement pour répondre à des demandes de l'environnement externe.

Ces exemples interprétatifs montrent à quel point la question des diverses influences sur les pratiques des enseignants est riche et complexe. Comme nous le disent Lessard, Altet, Paquay et Perrenoud (2004), les savoirs professionnels des enseignants ne constituent pas un corps de connaissances homogène et stabilisé.

Les récents travaux de Vause (2009) ajoutent également que les savoirs des enseignants se construisent grâce à leurs expériences en classe à l'aide de processus d'assimilation et d'accommodation. En s'inspirant du courant constructiviste piagétien, l'auteure postule que l'enseignement est influencé par le vécu scolaire personnel de l'individu. Dès lors, en plus des références épistémologiques propres à la discipline, il existe 
également une tendance à considérer l'épistémologie personnelle des enseignants. Hofer (2004) définit l'épistémologie personnelle comme un champ de recherche qui s'intéresse aux croyances et aux théories que les individus développent à propos des connaissances et de leur acquisition. La question de savoir comment ces croyances épistémiques peuvent influencer les processus cognitifs de pensée et de raisonnement est également au cœur des préoccupations de Berthiaume (2007). Ce chercheur propose, à ce titre, une redéfinition du DPK de Lenze (1995) en intégrant la dimension de l'épistémologie personnelle aux dimensions disciplinaires et pédagogiques. Il propose un modèle-outil qui permettrait de comprendre, voire d'interpréter toute l'hétérogénéité dont il a été question ci-dessus.

\subsection{Le savoir pédagogique disciplinaire -SPD}

Le projet de recherche de Berthiaume (2007) examine de façon empirique la nature du savoir pédagogique disciplinaire (SPD), savoir relié à l'enseignement d'une discipline à l'université. Comme nous l'avons vu précédemment, dans la tradition anglo-saxonne de recherches en éducation, le SPD trouve son origine dans deux branches de recherches : soit celles portant sur la base de connaissances pédagogiques, soit celles portant sur la spécificité disciplinaire. Mais, selon l'auteur, ces deux branches de recherches en éducation ne parviennent pas tout à fait à cerner le concept de SPD dans toute sa complexité. C'est la raison pour laquelle Berthiaume ajoute une nouvelle branche de recherches : celles qui touchent aux croyances à l'égard du savoir.

En effet, les recherches portant sur la base de connaissances pédagogiques (Hiebert, Gallimore \& Stigler, 2002 ; Munby, Russell \& Martin, 2001 ; Shulman, 1986) ne se prêtent pas facilement à un examen des facteurs qui seraient externes à la base de connaissances du professeur, telles que les normes, règles ou pratiques qui prévalent au sein d'une discipline universitaire.

Parallèlement, les recherches portant sur la spécificité disciplinaire (Becher \& Trowler, 2001 ; Donald, 2002 ; Hativa \& Marincovich, 1995 ; Neumann, 2001) ne permettent pas facilement d'expliquer des facteurs internes telles que les relations pouvant exister entre les divers types de connaissances ou les intentions du professeur.

Il apparaissait donc pour Berthiaume (2006) que les façons antérieures de conceptualiser le SPD, prises individuellement, limitaient les possibilités de comprendre le phénomène en question. Dès lors, à l'instar de Shulman (1986) et de Lenze (1995), il a réuni ces deux branches de recherches, ceci, parce que « la jonction de concepts provenant des recherches sur la base de connaissances pédagogiques et de recherches sur la spécificité disciplinaire fournit une façon de considérer simultanément les facteurs internes et externes contribuant à la formation de SPD » (Berthiaume, 2007, p.179). Or, il va encore plus loin en incluant des concepts provenant d'une troisième branche de recherches, soit celle portant sur les épistémologies personnelles, c'est-à-dire les croyances entretenues à l'égard du savoir (Hofer \& Pintrich, 1997, 2002) :

Cette dimension est essentielle à l'articulation du lien entre la base de connaissances pédagogiques et la spécificité disciplinaire puisque les croyances qui sont présentes dans la base de connaissances pédagogiques interagissent avec les savoirs propres à la discipline du professeur. Les épistémologies personnelles servent donc des fonctions de médiation entre les normes sociales d'une discipline 
et les pratiques pédagogiques d'un professeur enseignant cette discipline

(Berthiaume, 2007, p.179). génériques (son savoir pour enseigner) aux caractéristiques spécifiques de sa discipline (son savoir à enseigner) en tenant compte de son épistémologie personnelle (ses croyances). Berthiaume a testé le modèle-outil dans une étude multicas effectuée auprès de quatre professeurs provenant de quatre disciplines différentes. Sur la base d'éléments communs et des différentes relations entre ces éléments, il a constitué le noyau du SPD (repris dans la figure 2) ou les aspects du savoir pédagogique disciplinaire qui sont susceptibles d'être présents chez la plupart des professeurs.

Figure 2. Le savoir pédagogique disciplinaire selon Berthiaume (2006).

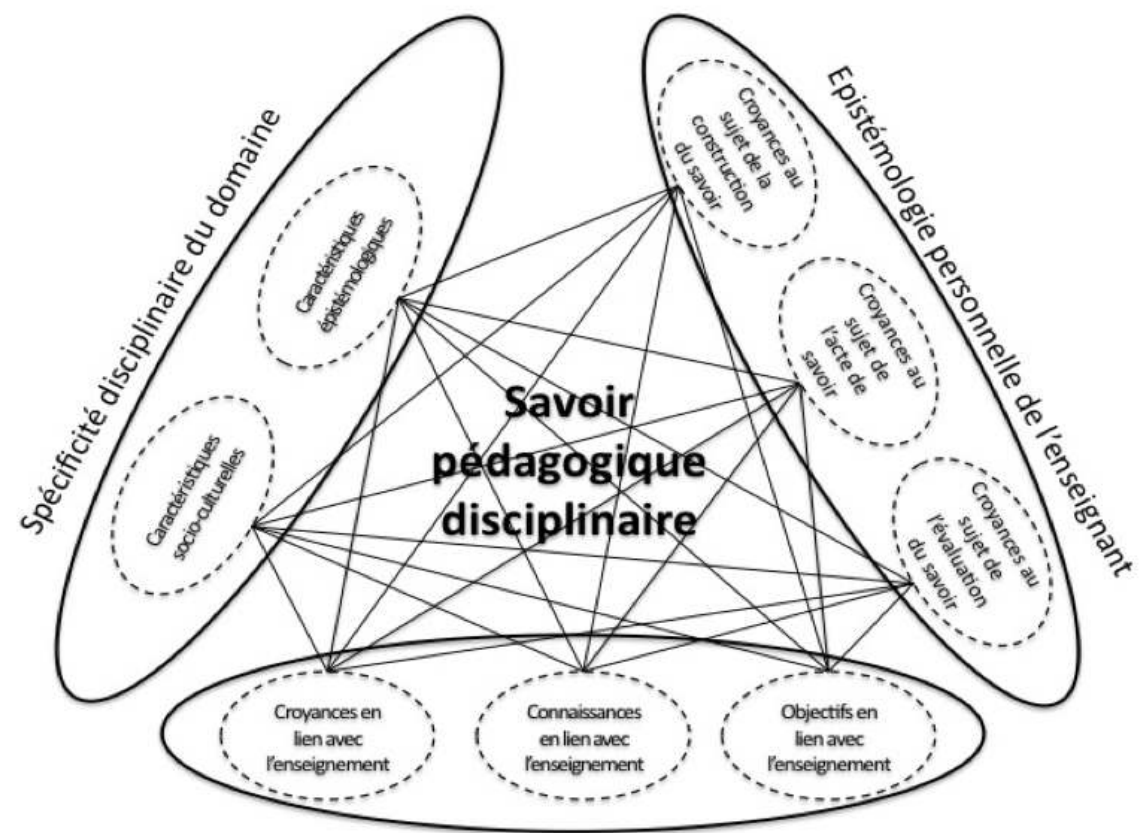

Base de connaissances pour l'enseignement

Ensuite, pour chaque cas de son analyse, il a identifié les relations spécifiques entre les composantes du modèle, celles-ci pouvant être expliquées par des ancrages disciplinaires différents. Globalement, nous pouvons dire que le projet de recherche de 
Berthiaume (2006) a produit un modèle-outil qui capture le phénomène du savoir pédagogique disciplinaire (SPD) des professeurs d'université de façon plus précise que les conceptualisations antérieures.

29 Le SPD de Berthiaume $(2006,2007)$ n'intègre pas la dimension technologique qui nous intéresse. À nous donc de compléter son modèle par l'apport de la dimension technologique étudiée dans un nouveau modèle qui se base également sur les réflexions de Shulman : le TPACK.

\section{Pédagogie, contenu et technologie}

En parallèle aux développements théoriques de Berthiaume (2007), un autre élargissement au modèle de Shulman a été proposé par Mishra et Koehler (2006). Ils invitent à associer au duo connaissances pédagogiques et connaissances disciplinaires, la dimension des connaissances sur les technologies. Leur modèle porte le nom de Technological and Pedagogical Content Knowledge(TPACK).

\subsection{Le technological and pedagogical content knowledge - TPACK}

31 Mishra et Koehler (2006) invitent à associer à ce duo la dimension des connaissances sur les technologies qui comprennent les connaissances sur l'usage d'outils technologiques tels que le tableau blanc interactif, internet ou encore les podcasts mais aussi les capacités à installer des périphériques ou à gérer un ordinateur (installer un programme, utiliser les logiciels, etc.).

Cette troisième dimension vient s'ajouter aux deux précédentes pour mieux encore rendre compte du fonctionnement de l'enseignement. Selon eux, la notion de TPCK ou TPACK (connaissance technopédagogique du contenu) est complètement nouvelle. L'approche plus spécifique du TPACK consiste à analyser les relations entre contenu, pédagogie et technologie. En plus de considérer chacune de ces entités de manière séparée, cela signifie qu'il y a une étude systématique sur les interrelations par paires entre les connaissances pédagogiques du contenu (association des connaissances pédagogiques et des connaissances du contenu), les connaissances technologiques du contenu (association des connaissances technologiques et des connaissances du contenu), les connaissances technopédagogiques (association des connaissances technologiques et des connaissances pédagogiques) et l'intersection de ces trois dimensions : les connaissances technopédagogiques du contenu. 
Figure 3. Modèle TPACK adapté de Mishra et Koehler (2006).

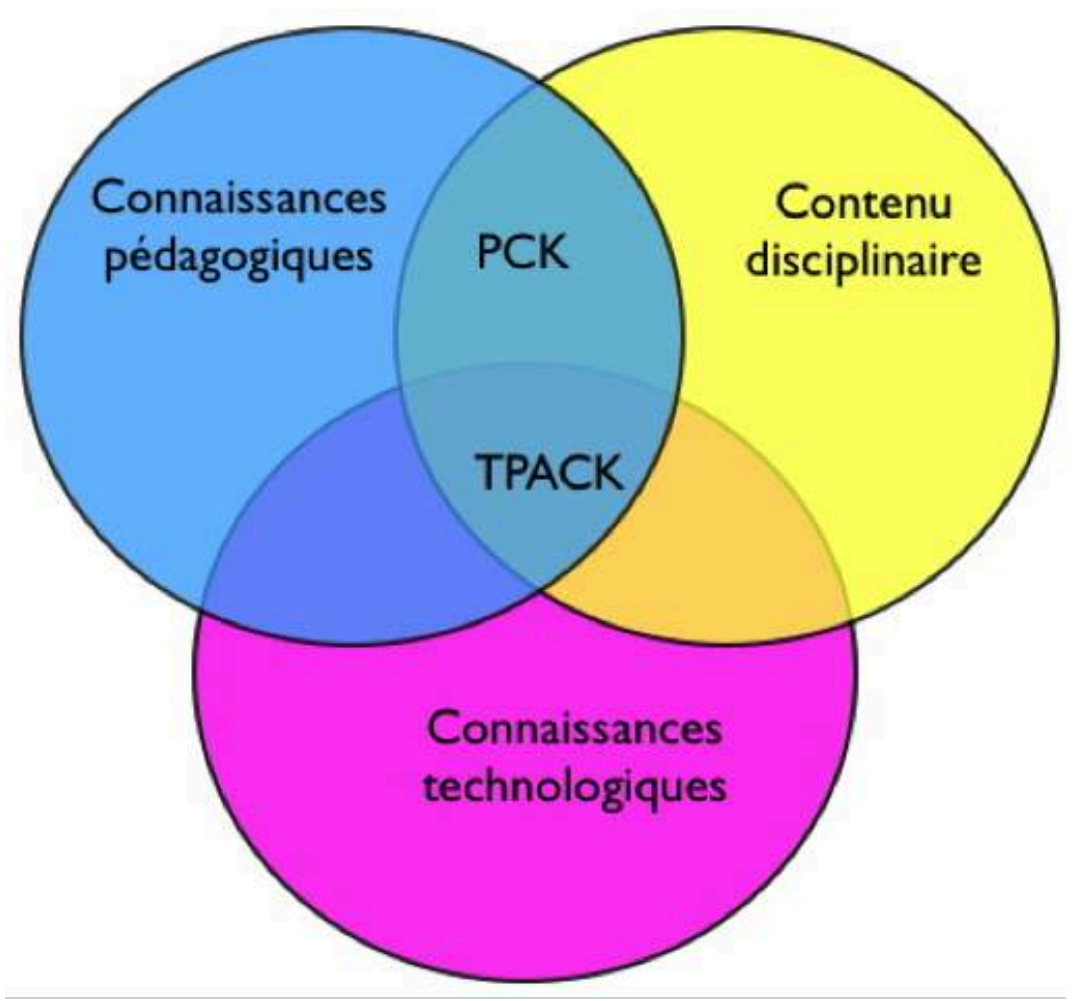

Nous avions déjà expliqué plus haut la première intersection qui concerne les connaissances pédagogiques du contenu. Voyons à quoi correspondent les deux autres. Les connaissances technologiques du contenu concernent la relation entre les technologies et un contenu. Il s'agit, ici, de savoir comment les technologies peuvent influencer l'objet à enseigner (Mishra \& Koehler, 2006). Ils prennent l'exemple de l'enseignement de la géométrie. L'usage d'un carnet de croquis virtuels permet aux apprenants de tester très facilement plusieurs combinaisons sans devoir passer par l'obligation de construire chaque élément pour en vérifier les proportions. De ce fait, cela change la nature de l'apprentissage en lui-même (Niess, 2005). Les connaissances technopédagogiques concernent toutes les connaissances des différentes technologies pour enseigner et apprendre. Il s'agit des capacités à pouvoir associer certains outils pour des tâches pédagogiques spécifiques. Et l'intersection de ces trois dimensions forme le TPACK.

Pour Mishra et Koehler (2006), les connaissances technopédagogiques du contenu qui est une nouvelle forme de connaissances avec :

- La base d'un bon enseignement avec les technologies et qui nécessite une compréhension de la représentation des concepts en utilisant les technologies;

- L'utilisation de techniques pédagogiques qui utilisent les technologies de manière constructive pour enseigner un contenu ;

- La connaissance de ce qui rend des concepts difficiles ou simples à apprendre et comment les technologies peuvent aider à résoudre une partie du problème que l'apprenant rencontre ;

- La connaissance sur la manière dont les technologies peuvent être utilisées pour construire un savoir sur des bases existantes et en développer de nouvelles ou modifier les anciennes. 

dimensions (pédagogie, contenu ou technologie) sont indissociables. Le changement d'une des trois dimensions devrait être compensé par un changement dans les deux autres. Pour illustrer ce propos, prenons un cours en sciences politiques qui propose des analyses de cas sur la base de cartes géographiques illustrées qui sont projetées au cours. Des effets dynamiques montrent des enjeux géostratégiques. Par la présence même de ces outils technologiques (PowerPoint animé) l'enseignant vise pédagogiquement des études de cas pour faire construire des connaissances en géopolitique. Si maintenant son objectif était uniquement de transmettre les faits historiques sans pointer ni analyser les enjeux, il pourrait se contenter de les donner oralement sans support visuel ou sans animation.

Le TPACK est un modèle qui défend l'idée d'interrelations fortes entre contenu, pédagogie et technologie, intégrant le contenu dans une dynamique à double sens. Les enseignants ne sont pas obligés d'utiliser les trois composantes, mais les choix qu'ils font auront des répercussions sur le système. Les interrelations ont été validées par de nombreux travaux comme ceux de Archambault et Crippen (2009) ou Schmidt, Baran, Thompson, Mishra, Koehler et Shin (2009).

Selon Mishra et Koehler (2006), l'apparition récente des technologies en ligne force les enseignants à trouver un équilibre entre ces trois dimensions souvent les conduisant à se questionner à propos de leur pédagogie. Les études de Milken family Foundation and the international society for Technology (1999) montrent à ce propos que les programmes actuels de formation des enseignants ne leur fournissent pas les expériences nécessaires pour qu'ils puissent utiliser les technologies. L'enquête montre que les apports théoriques sur les technologies n'ont quasi pas d'impact sur leur utilisation dans les pratiques des enseignants. Pour suivre l'une des recommandations de leurs travaux, Mishra et Koehler (2006) préconisent d'apprendre par la conception. Ils sont à l'origine de toute une série d'applications que nous proposons d'analyser ci-dessous.

\subsection{Application dans les formations pédagogiques}

38 Afin de mieux supporter l'impact des formations en e-learning sur les pratiques des enseignants, Mishra et Koehler (2006) ont analysé la portée de leur modèle TPACK. Ils défendent l'idée qu'il faut apprendre par l'action. Le « learning by doing » se situe entre la théorie et la pratique. Les enseignants sont confrontés à des situations où ils doivent opérer des choix stratégiques comme en situation réelle. Ainsi, les auteurs vont jusqu'à parler d'inférence de leur théorie c'est-à-dire que la théorie du TPACK permet non seulement de comprendre ce qu'enseigner avec les technologies signifie, mais aussi de faire des prédictions et d'inférer en fonction de contexte de ce que pourrait être un enseignement de qualité. Le TPACK peut être une structure organisationnelle utile pour définir ce que doivent savoir les enseignants pour intégrer les technologies dans leur enseignement (Archambault \& Crippen, 2009).

Dès lors, de nombreuses recherches ont ciblé leurs démarches sur les applications possibles dans les formations des enseignants. Niess (2005) a mis en place un protocole de formation technopédagogique pour des enseignants en sciences et mathématiques. Dans son étude, l'auteur analyse le changement de profil des enseignants au niveau du TPACK. Hofer et Harris (2010) lui reprochent son caractère trop généraliste. Ils ont ainsi analysé que la plupart des applications proposées sur la base du TPACK se focalisaient,

Revue internationale de pédagogie de l'enseignement supérieur, 30(2) | 2014 
en premier lieu, sur les outils et les ressources technologiques. À juste titre, ils évoquent que ce genre de formation ne peut pas tenir compte de l'hétérogénéité des profils des enseignants. Ils ont, dès lors, mis au point des activités types d'apprentissage(Learning Activity Type) pour accompagner les enseignants à mettre en relation leurs objectifs d'enseignement basés sur le curriculum, les activités pédagogiques et les outils technologiques. En pratiquant de la sorte, ils rejoignent Shulman (1986) et Mishra et Koehler (2006) pour considérer que les pratiques d'enseignement (et donc le choix des outils technologiques) varient en fonction des contenus d'enseignement. Comme nous le verrons plus loin, cette démarche correspond à celle qui a été adoptée dans notre recherche pour guider les enseignants lors de la scénarisation. Elle correspond également aux travaux de Milken (1999) et de Puentedura (2006) qui avaient analysé les différents usages des technologies par rapport aux différents domaines précités.

Hofer et Harris (2010) proposent d'élaborer une scénarisation qui tient compte des objectifs pédagogiques, des styles d'apprentissage (principe de variation des activités proposées), d'une certaine cohérence dans les séquences d'apprentissage, d'une évaluation formative et d'une sélection de ressources et d'outils qui peuvent aider les apprenants. Selon eux, cette approche permet de se décentrer du contenu à enseigner (tendance classique chez les enseignants débutants) pour se focaliser sur l'apprentissage des étudiants. De plus, avec cette stratégie, les enseignants deviennent davantage conscients de ce qu'ils mettent en place et les activités deviennent plus centrées sur l'apprentissage (Graham, Borup \& Smith, 2012).

41 Les instruments publiés pour le TPACK pour valider les changements possibles dans les pratiques d'enseignement ne sont que d'un seul type : des tests d'auto-positionnement. Schmidt et al. (2009) ont ainsi développé un instrument d'auto-positionnement avec de nombreux items se rapportant aux différentes dimensions du TPACK : technological $(\mathrm{T})$, pedagogical (P), content $(\mathrm{C})$, technological pedagogical (TP), technological content (TC), pedagogical content (PC) et technological pedagogical content knowledge (TPACK).

Schmidt et al. (2009) examinent comment de futurs enseignants établissent les relations entre ces sept dimensions durant leur formation de base. Archambault et Crippen (2009) ont également développé un test de ce type plutôt destiné aux enseignants déjà en fonction. Harris, Grandgenett et Hofer (2010) reconnaissent que ces deux tests apportent une aide considérable pour évaluer le profil TPACK des enseignants qui s'auto-positionnent sur la base d'une échelle de compétence à cinq niveaux. Toutefois, ces informations doivent être recoupées avec des évaluations externes de leur enseignement. Alors, ils proposent une grille d'observation avec des critères assez semblable aux échelles de Milken (1999). Harris, Grandgenett et Stigler (2010) ajoutent un critère de cohérence entre les objectifs poursuivis (en fonction du curriculum), les stratégies pédagogiques et les choix technologiques. Pour chacun des critères, ils proposent des indicateurs qui démontrent un niveau débutant (par exemple, les objectifs pédagogiques et les choix technologiques ne sont pas alignés) ou à l'inverse un niveau avancé (les choix technologiques sont alignés avec les objectifs pédagogiques). 


\section{Le modèle du savoir technopédagogique disciplinaire}

À partir des analyses des séquences d'enseignement-apprentissage en ligne et des tentatives pour mieux comprendre comment les enseignants établissent leurs choix pédagogiques, nous avons intégré les idées des modèles PCK, DPK, SPD et TPACK. Par leur combinaison, nous pouvons interroger les enseignants sur les liens qu'ils font entre leurs connaissances pédagogiques (P), leurs connaissances technologiques (T), leur épistémologie personnelle (E) et leur discipline (D).

Les travaux de Lenze (1995) justifient le remplacement du «content» du PCK par « discipline-specific» dans le DPK parce qu'à l'université, le contenu correspond aux disciplines académiques.

Dans les modèles PCK et TPACK, les contenus sont expliqués en fonction des disciplines et dans le modèle SPD la dimension discipline fait référence à la structure épistémologique et aux caractéristiques socioculturelles pour enseigner un contenu. Le modèle STPD vise bien à comprendre les relations que font les enseignants entre ces dimensions ( $\mathrm{T}, \mathrm{P}, \mathrm{E}$ et $\mathrm{D}$ ) pour enseigner un contenu issu du champ disciplinaire de l'enseignant.

Figure 4. Savoir technopédagogique disciplinaire - STPD (Bachy, 2013).

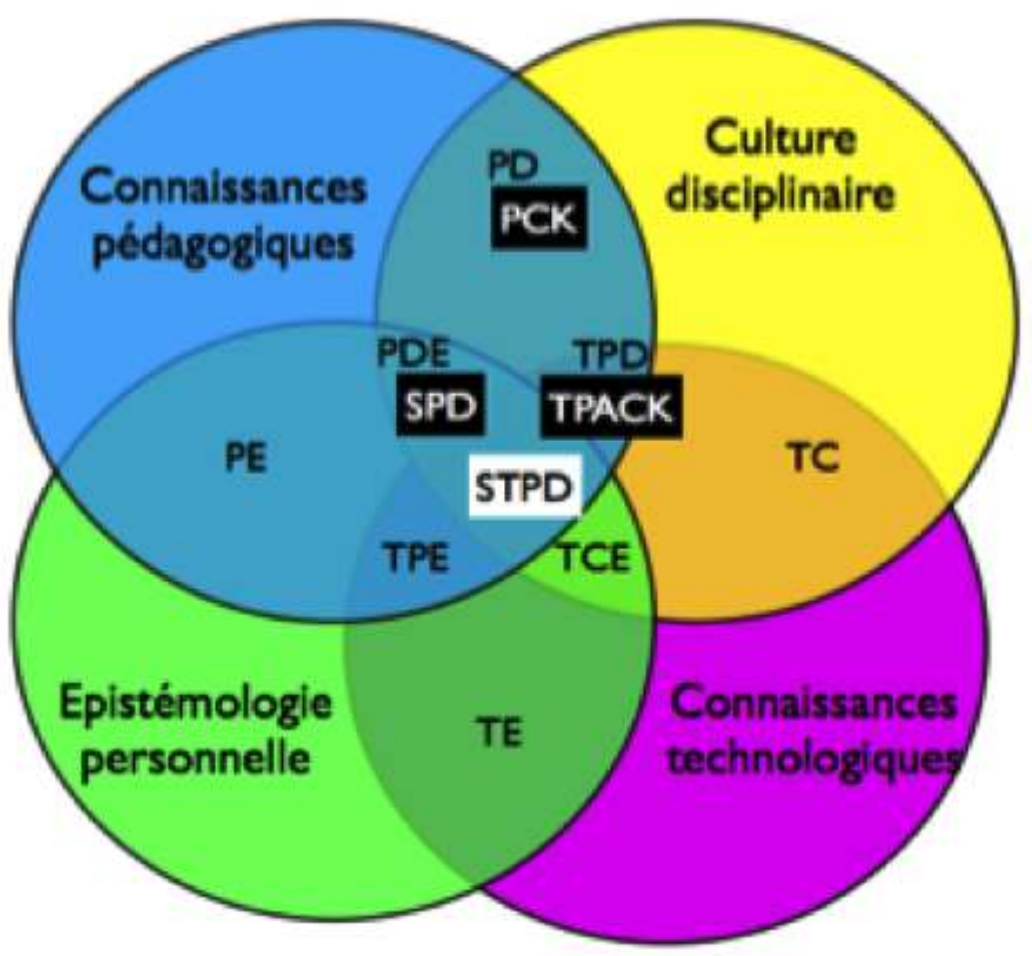

Les six relations entre les quatre dimensions du STPD sont représentées ci-après (figure 5). Nous avons :

- PE : la relation entre les connaissances pédagogiques et l'épistémologie personnelle ;

- PD : la relation entre les connaissances pédagogiques et la discipline (ce qui correspond au PCK de Shulman ou DPK de Lenze) ; 
- TP : la relation entre les connaissances pédagogiques et les connaissances technologiques ;

- TE : la relation entre les connaissances technologiques et l'épistémologie personnelle ;

- TD : la relation entre les connaissances technologiques et la discipline ;

- DE : la relation entre la discipline et l'épistémologie personnelle.

Figure 5. Relations entre les dimensions du STPD.

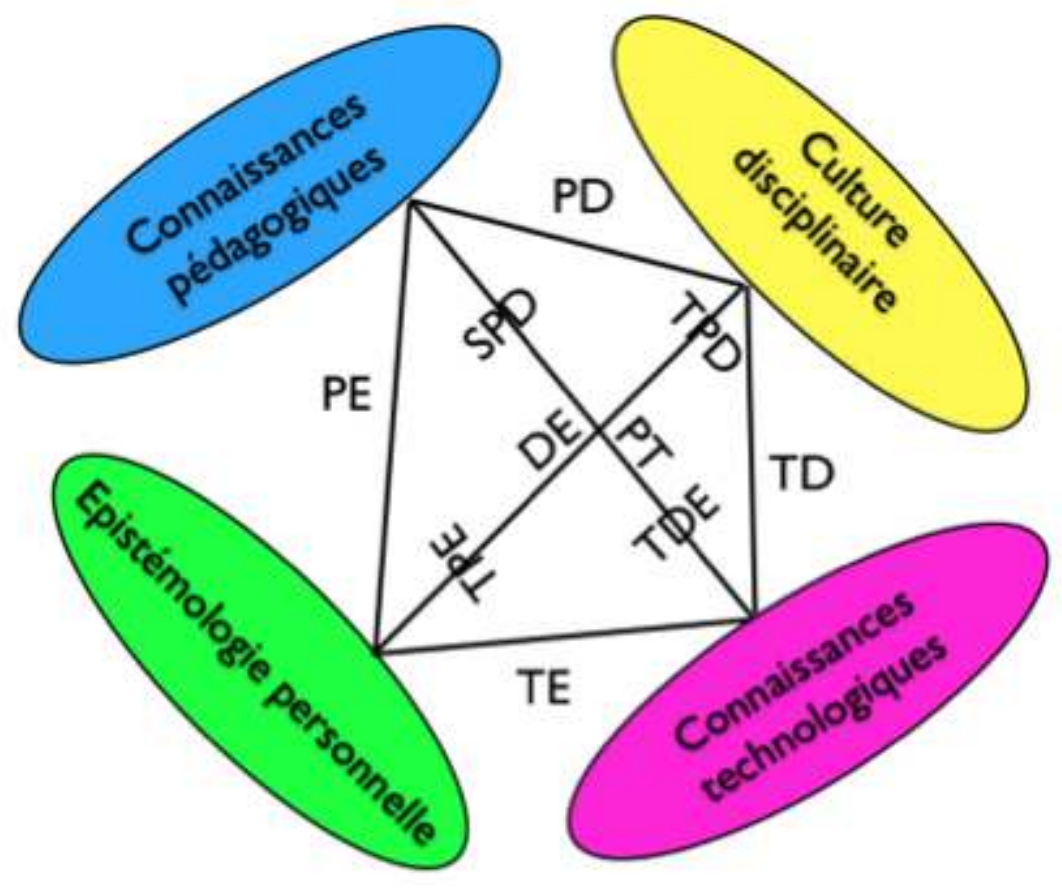

Dans ce qui suit, nous allons analyser la pertinence de l'intégration des quatre dimensions puis nous terminerons par une discussion sur les perspectives d'usage pour les conseillers pédagogiques et les perspectives de recherche pour la formation des enseignants.

\section{Méthodologie}

Sur la base des questionnaires élaborés dans les travaux d'Archambault et Crippen (2009) et de Schmidt et al. (2009), notre questionnaire d'auto-positionnement s'emploie à tester chaque dimension et les relations entre elles. Le public visé par le test d'Archambault et Crippen (2009) étant déjà en responsabilités d'enseignement par opposition aux élèves-enseignants visés par Schmidt et al. (2009), nous avons davantage pris leurs items comme modèles. Pour chaque dimension et chaque relation, le test d'Archambault et Crippen proposait trois ou quatre items. Au total, les auteurs ont testé les compétences technopédagogiques des enseignants avec 24 items. Ils ont soumis leur test à 576 enseignants impliqués dans des classes virtuelles de 25 états des États-Unis. Les enseignants devaient estimer différentes capacités sur la base de cinq niveaux : pauvre, satisfaisant, bon, très bon et excellent. Ces auteurs ont trouvé des corrélations positives entre toutes les dimensions dont certaines étaient fortement significatives (Archambault \& Crippen, 2009, p.80). 
49 Notre questionnaire s'appuie partiellement sur les items qu'ils ont proposés et nous avons ajouté la dimension de l'épistémologie personnelle (E) et les relations avec les technologies (TE), la pédagogie (PE) et la discipline (DE). Nous avons élaboré trois items pour les 10 catégories. Comme nous le verrons plus loin au moment de la validation, parmi les 30 items initiaux, nous avons dû en retirer deux pour assurer une meilleure cohérence interne (l'alpha de Cronbach était trop faible pour l'élément testé). Tous les items sont repris ci-après (tableau 1 ). Les items $1,2,3,4,5,6,7,9,19,20$ et 21 proviennent d'une traduction libre des items proposés par Archambault et Crippen (2009). Ils ont fait l'objet d'une confrontation avec d'autres théories qui traitent des disciplines, des connaissances pédagogiques et des connaissances technologiques. Ceux qui n'ont pas été traduits du test TPACK initial ne mettaient pas, selon nous, une dimension suffisamment en valeur. Par exemple, le test TPACK prévoit un item «My ability to distinguish between correct and incorrect problem solving attempts by students " pour tester la relation Pedagogical Content Knowledge. Comme nous avons traduit le terme contenu en discipline pour intégrer le modèle SPD et adapter le TPACK aux enseignants de l'université, cet item ne met pas suffisamment l'accent, selon nous, sur la discipline. Nous avons, dès lors, formulé de nouveaux items sur la base de notre interprétation des composantes du SPD de Berthiaume (2007).

50 Ainsi, les six relations (PE, TE, TD, TP, PD, TE) et les quatre dimensions (T, D, P et E) sont chacune testées par deux ou trois items. Les enseignants ont été invités à répondre à la question "Comment évaluez-vous vos capacités à réaliser les tâches présentées cidessous, liées à l'enseignement?». Les réponses sont notées au moyen d'une échelle de Likert qui comprend cinq niveaux $(1=$ pauvre, $2=$ satisfaisant, $3=$ suffisant, $4=$ bon et 5 = excellent). Nous n'avons pas procédé à la validation d'items pouvant regrouper trois dimensions (PDE, TDE, TPD et TPE). En effet, nous avons trouvé que la formulation de ces items était très complexe et que, par ailleurs, nous disposions assez d'éléments pour pouvoir regarder les relations existantes. 
Tableau 1. Items du STPD où les items 4 et 11 ont été barrés suite à l'analyse de cohérence

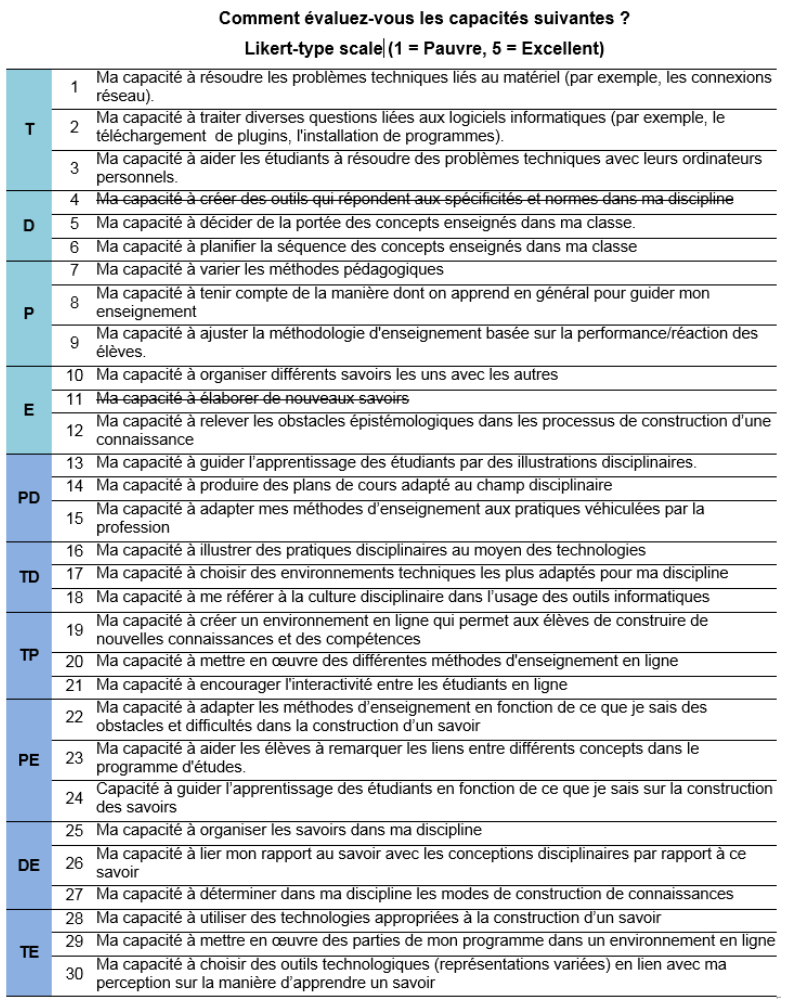

51 Pour chaque item des dimensions testées, les enseignants devaient donner une valeur comprise entre 0 et 5 qui correspond à leur sentiment de compétence. Pour dresser leur profil sous la forme d'un radar, nous avons calculé la moyenne des valeurs par dimension. Pour nous assurer de la pertinence des regroupements des items par dimension, nous avons réalisé un test de cohérence (alpha de Cronbach). En guise de pré-test nous avons également voulu vérifier la sensibilité de la composante technologique avec un groupe pilote d'enseignants montrant des compétences technologiques différentes.

\section{Validation}

Nous avons procédé en deux étapes à la validation du questionnaire, premièrement, par une analyse de validité avec un groupe pilote et, deuxièmement, par une analyse de cohérence des items par dimension. Nous proposons une analyse des coefficients de corrélation linéaire de Bravais-Pearson pour tester les relations entre ces dimensions.

\subsection{Analyse de validité avec un groupe pilote}

Pour nous assurer que les items mesurent bien ce qu'ils doivent mesurer et qu'ils sont exploitables en l'état, nous avons fait passer la grille à quatre enseignants du niveau universitaire. Nous connaissions bien leurs profils technologiques et pédagogiques pour les avoir consultés, à de nombreuses reprises, à propos de leurs dispositifs d'enseignement. Nous avons délibérément choisi quatre profils très différents par rapport à l'usage des technologies. Ce groupe pilote a été sélectionné pour vérifier la 
construction des items (sont-ils clairs et bien compris ?) et la portée des variations de profils observables avec une construction de radars. Ce groupe comprend:

- Le professeur A : professeur en langues germaniques pour les ingénieurs de gestion depuis 15 ans. Il n'utilise pas les technologies sauf lorsqu'il y est obligé dans le cadre de ses collaborations avec d'autres enseignants (utilisation des mêmes supports de cours pour tous les enseignants de l'unité). Il n'a pas de cours en ligne. Il n'a pas suivi de formations liées aux technologies. Il a déjà participé à des formations pédagogiques ;

- Le professeur B : enseignant à l'école des ingénieurs depuis 12 ans. Il n'a pas de cours en ligne et n'a jamais suivi de formations liées aux technologies. Par contre, il donne des formations pédagogiques. Pour ses cours, il utilise des diaporamas, de nombreuses illustrations et il enregistre des podcasts de son cours dans leur intégralité (sans montage) ;

- Le professeur $\mathrm{C}$ : enseignant en sciences politiques depuis 7 ans. Il commence à enseigner en ligne (un an d'expérience). Il a déjà suivi une formation liée à l'e-learning. Il a appris à scénariser ses cours (voir à ce propos la méthode proposée dans l'article Collès et Bachy 2011) ;

- L'assistant D enseigne les statistiques depuis 8 ans. Il a plusieurs cours en ligne. Il a suivi des formations pédagogiques et technologiques. Il vient de terminer un master en pédagogie universitaire. Il développe des interfaces informatiques spécifiques pour guider l'apprentissage des étudiants. Il s'intéresse aux obstacles épistémologiques dans l'apprentissage.

Les quatre enseignants ont pu répondre à presque tous les items. Parfois, une hésitation pour les items $(16,18,24$ et 28$)$, mais sans que cela ne porte à conséquence sur les réponses. Le professeur $\mathrm{B}$ a eu plus de mal que les autres à comprendre le concept "technologie» dans les items. Ce concept est également utilisé chez les ingénieurs sous un autre sens.

L'analyse en rang 1 (figure 6) permet de voir quelle est la part de la dimension technologique dans le profil des enseignants par rapport aux autres dimensions. A et D présentent deux profils bien contrastés. Ils estiment tous les deux avoir de bonnes compétences disciplinaires, pédagogiques et épistémologiques, mais A n'en a pas pour les technologies alors que $\mathrm{D}$ se place à un niveau d'excellence dans sa maitrise des technologies.

Le rang 2 permet de faire émerger certaines remarques. Par exemple, l'enseignant B fait davantage de liens entre les composantes discipline et la manière dont il perçoit que l'on construit un savoir (DE). De même, la façon dont il perçoit la construction de certains contenus influence fortement ses actions pédagogiques (PE). L'ancrage épistémologique (les croyances à propos des savoirs et des manières de les apprendre) domine dans son profil. On voit qu'il utilise les technologies, mais il ne les associe pas nécessairement pour prendre des décisions pédagogiques. 
Figure 6. Analyse de validité avec un groupe pilote.

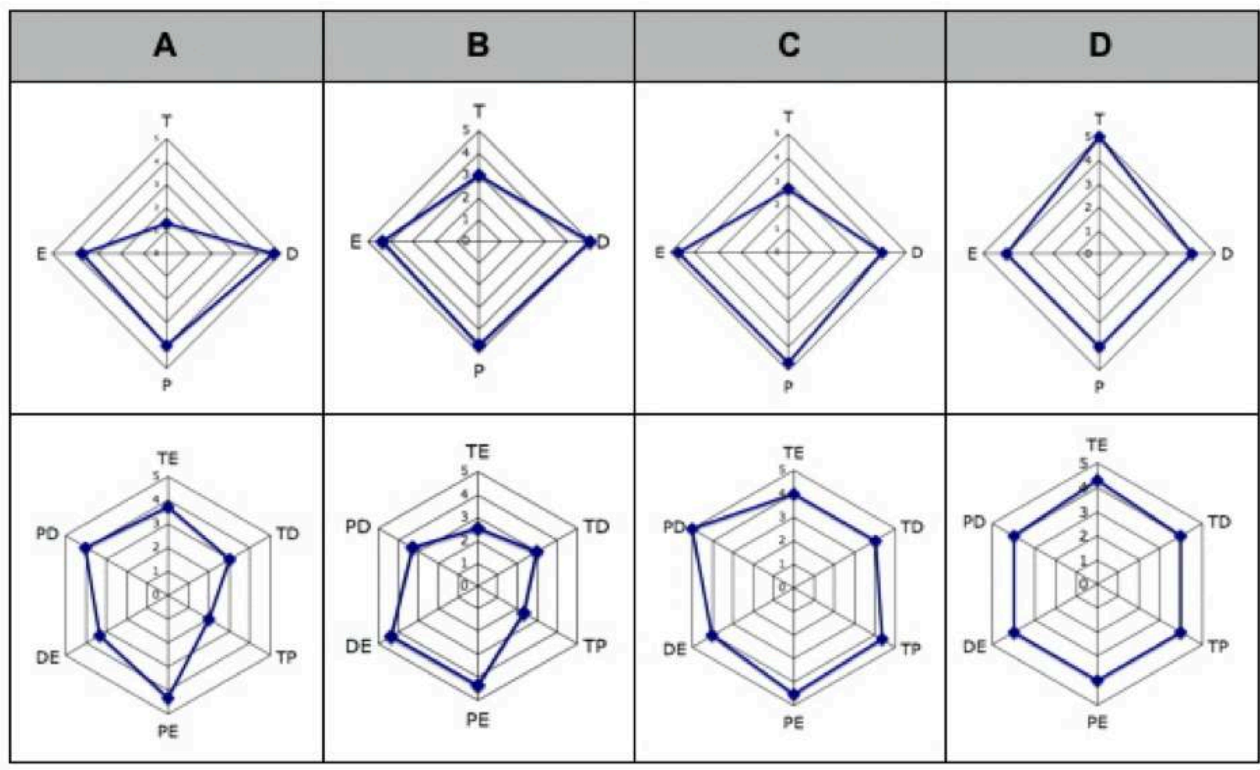

57 Le profil de $C$, qui montrait un même sentiment de compétence dans ses capacités technologiques que $\mathrm{B}$, montre un autre fonctionnement. Il fait beaucoup plus de liens entre la dimension technologique et la pédagogie (TP). Des quatre enseignants interrogés, c'est le seul qui a suivi une démarche de scénarisation et une formation elearning.

Les profils STPD élaborés grâce au questionnaire de 28 items semblent bien correspondre à ce que l'on sait de ces enseignants par rapport à leurs connaissances technologiques et par rapport à la justification qu'ils font de leurs activités pédagogiques.

\subsection{Analyse de cohérence}

Conformément aux recommandations de Czaja et Blair (2005) nous avons mis le questionnaire sur LimeSurvey et nous l'avons soumis à 150 enseignants de l'enseignement supérieur universitaire et non universitaire dans le cadre d'un autre projet de recherche qui était en cours. Un alpha de Cronbach a été calculé pour chaque dimension pour nous assurer que le regroupement des items était bien cohérent au sein des dimensions. Sur la base des réponses $(\mathrm{N}=55)$, nous avons retiré deux items (Item 4 : Ma capacité à créer des outils qui répondent aux spécificités et normes dans ma discipline et Item 11 : Ma capacité à élaborer de nouveaux savoirs) pour que la dimension soit la plus proche possible de 0,71 limite de fiabilité avec l'indice de Cronbach. Pour la relation DE, l'indice reste faible $(a l p h a=0,639)$ il est toutefois présenté ici avec sa valeur de fiabilité la plus haute. 
Tableau 2. Alpha de Cronbach

\begin{tabular}{|c|c|c|c|}
\hline Domaine & $\begin{array}{c}\text { Nombre d'items par } \\
\text { dimension }\end{array}$ & $\begin{array}{c}\text { Nombre de réponses valides } \\
(\mathbf{N}=55)\end{array}$ & $\begin{array}{c}\text { Alpha de } \\
\text { Cronbach }\end{array}$ \\
\hline Technologie (T) & 3 & 52 & 0,923 \\
\hline Discipline (D) & 2 & 35 & 0,760 \\
\hline Pédagogie (P) & 3 & 51 & 0,799 \\
\hline $\begin{array}{c}\text { Épistémologie } \\
\text { (E) }\end{array}$ & 2 & 33 & 0,737 \\
\hline PD & 3 & 25 & 0,703 \\
\hline TD & 3 & 38 & 0,878 \\
\hline TP & 3 & 42 & 0,872 \\
\hline PE & 3 & 40 & 0,745 \\
\hline DE & $3 E$ & 27 & 0,891 \\
\hline \hline
\end{tabular}

Pour les autres dimensions, les trois items élaborés montrent une bonne cohérence interne avec un alpha proche ou supérieur à 0,71 .

\subsection{Analyse de corrélations entre les dimensions}

61 En fonction des moyennes des résultats pour les différentes dimensions, nous avons réalisé un test de Pearson (tableau 4). L'objectif était de valider l'idée de système et des liens entre les quatre dimensions de notre modèle. L'analyse des coefficients de corrélation linéaire de Bravais-Pearson montre quelques corrélations positives et significatives intéressantes. 
Tableau 3. Corrélation entre les dimensions

\begin{tabular}{|c|c|c|c|c|c|c|c|c|c|c|}
\hline & T & D & P & E & PD & TD & TP & PE & DE & TE \\
\hline T & - & & & & & & & & & \\
\hline D & 0,028 & - & & & & & & & & \\
\hline$P$ & $0,274^{\star}$ & $0,489^{\star \star}$ & - & & & & & & & \\
\hline E & 0,240 & $0,533^{\star \star}$ & $0,491^{\star \star}$ & - & & & & & & \\
\hline PD & 0,136 & 0,262 & $0,484^{\star \star}$ & $0,537^{\star \star}$ & - & & & & & \\
\hline TD & $0,641^{\star \star}$ & 0,194 & $0,359^{\star \star}$ & $0,315^{\star}$ & 0,242 & - & & & & \\
\hline TP & $0,559^{\star \star}$ & 0,175 & $0,482^{\star \star}$ & 0,232 & 0,232 & $0,658^{\star \star}$ & - & & & \\
\hline PE & 0,142 & $0,413^{\star \star}$ & $0,552^{\star \star}$ & $0,718^{\star \star}$ & $0,462^{\star \star}$ & $0,344^{\star}$ & 0,181 & - & & \\
\hline DE & $-0,54$ & $0,487^{\star \star}$ & $0,354^{\star}$ & $0,704^{\star \star}$ & $0,325^{\star}$ & 0,41 & $-0,50$ & $0,638^{\star \star}$ & - & \\
\hline TE & $0,528^{\star \star}$ & 0,267 & $0,483^{\star \star}$ & 0,282 & 0,177 & $0,694^{\star \star}$ & $0,773^{\star \star}$ & 0,200 & $-0,165$ & - \\
\hline
\end{tabular}

* Corrélation significative au niveau 0,05, ** corrélation significative au niveau 0,01.

62 En fonction du nombre de nos répondants $(\mathrm{N}=55)$ nous pouvons dire que la discipline est fortement corrélée de manière positive avec la pédagogie $\left(r=0,489^{* *}\right)$ et l'épistémologie $\left(r=0,533^{* *}\right)$. Autrement dit, d'après notre test, quand l'enseignant fait des choix disciplinaires c'est en lien avec ses croyances au niveau de la construction du savoir et ce qu'il sait de la pédagogie. Ces trois dimensions : P-D-E semblent généralement fortement liées de manière significative. Les travaux d'Archambault et Crippen (2009) montraient également de fortes corrélations positives entre contenu (Content) et pédagogie $\left(r=0,690^{* *}\right)$. L'ajout de la dimension épistémologique reprise dans travaux de Berthiaume (2007) semble pertinent lorsqu'on remarque les corrélations positives et significatives avec la discipline et la pédagogie.

En ce qui concerne la dimension des connaissances technologiques, elle apparait liée à la dimension pédagogique $\left(r=0,274^{*}\right)$. Par contre, elle n'est pas liée à la discipline $(r=$ 0,028) contrairement aux travaux d'Archambault et Crippen (2009) et elle est liée de manière non significative avec l'épistémologie personnelle. Par contre, les connaissances pédagogiques (tableau 4) sont corrélées de manière significative avec toutes les dimensions. Autrement dit, nous pourrions penser que les connaissances technologies n'ont de sens que si elles sont associées à des connaissances pédagogiques. Notons également que toutes les relations qui comprennent la dimension technologique : TE, TD et TP ne sont jamais corrélées de manière significative avec la discipline! Nous en rediscuterons plus loin. 


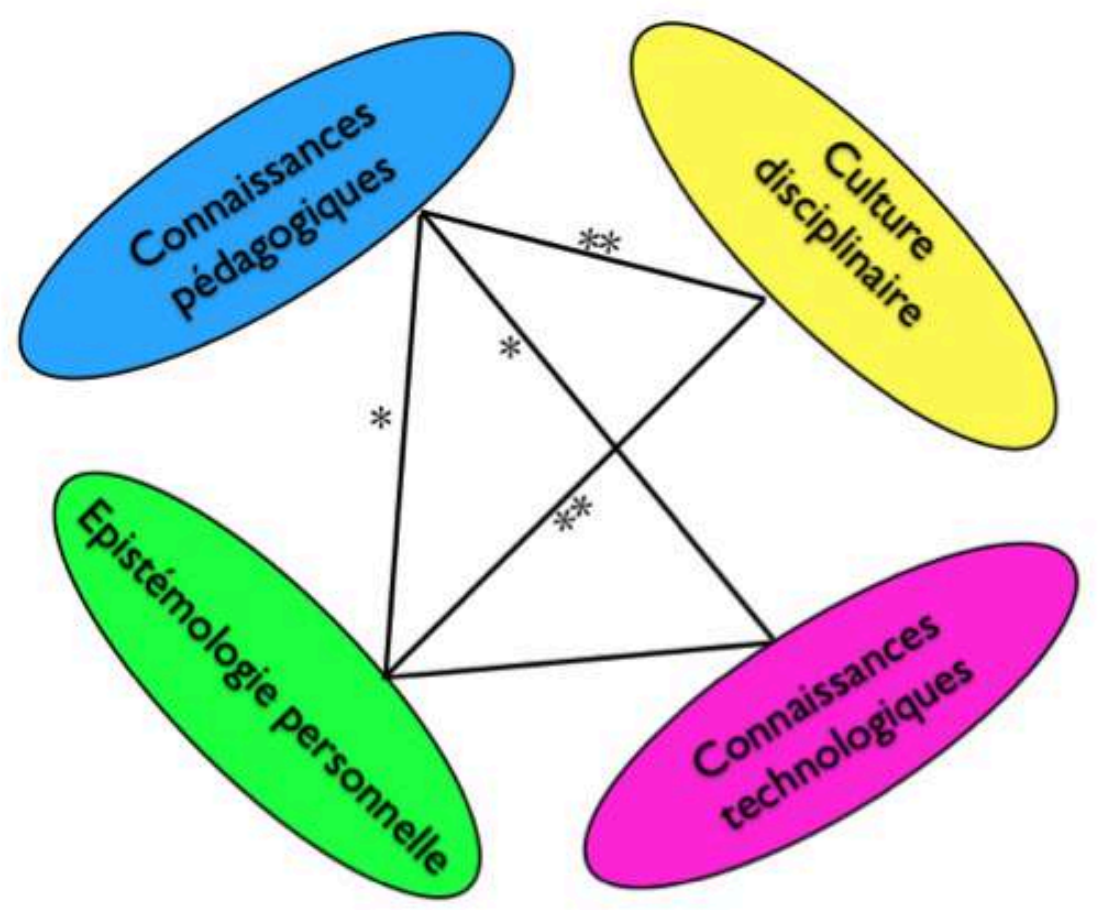

Les relations (figure 7) entre les quatre dimensions se présentent de manière à valider le modèle SPD (le noyau commun aux disciplines) de Berthiaume (2007) qui a mis en évidence les relations entre les connaissances pédagogiques, l'épistémologie personnelle et la discipline chez les enseignants de l'enseignement supérieur. Cela valide également les fondements des recherches de Shulman (1986) et de Lenze (1995) qui ont associé discipline et pédagogie. Enfin, cela permet également de valider le lien entre pédagogies et technologies, présent dans les travaux de Lebrun $(2002,2006$, 2007a, 2007b, 2012).

Par contre, nous ne trouvons pas de liens directs dans notre étude entre les connaissances technologiques et la discipline. Néanmoins, l'analyse des résultats (figure 8) montre des relations plus complexes. On voit que la discipline est davantage liée aux relations entre la pédagogie et l'épistémologie (PE), mais lorsqu'il y a présence des technologies associées à une autre dimension (TE, TD, TP) dans le profil des enseignants, il existe alors des liens significatifs avec la relation pédagogie-discipline (PD). Ainsi, on pourrait considérer que la relation entre connaissances technologiques et discipline serait peut-être médiée par l'épistémologie personnelle et/ou les connaissances pédagogiques. 
Figure 8. Corrélations entre les quatre dimensions et les relations entre ces dimensions.
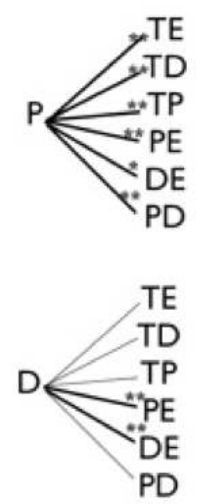
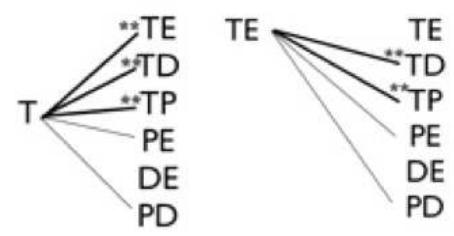

PE

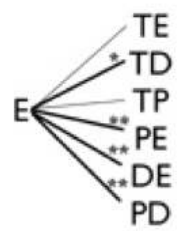

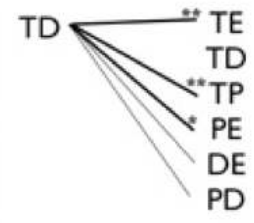

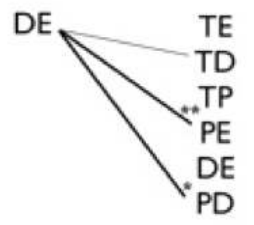

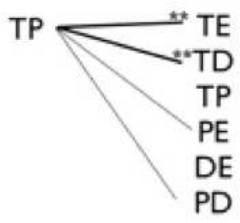

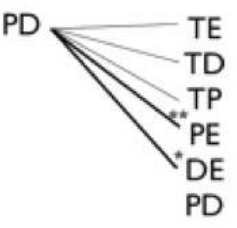

Quand un enseignant se sent compétent par rapport aux technologies dans sa discipline (dimension TD), cela influence ses choix pédagogiques $\left(r=0,359^{* *}\right)$ et, dans une moindre mesure, nous observons une corrélation plus faible (mais significative) avec ses choix épistémologiques $\left(r=0,315^{*}\right)$. Par ailleurs, si l'enseignant se sent compétent dans ses choix technologiques en fonction de ses croyances épistémologiques (TE), un lien assez fort se crée avec la dimension pédagogique $\left(\mathrm{r}=0,483^{* *}\right)$ mais également avec d'autres combinaisons comme TD $\left(r=0,694^{* *}\right)$ ou TP $\left(r=0,773^{* *}\right)$. La présence de la corrélation positive et significative entre PE et TD (soit les quatre dimensions) nous inviterait à penser que les dimensions sont bien en relation les unes par rapport aux autres, mais que la dimension $\mathrm{T}$ ne le serait que parce qu'elle est associée à une autre dimension. Les corrélations pourraient s'expliquer par le fait que nous avions trouvé des liens entre la dimension pédagogique et les autres dimensions.

À l'instar des travaux d'Archambault et Crippen (2009), nous trouvons une forte corrélation $\left(0,658^{* *}\right)$ entre les technologies-discipline (TD) et les technologiespédagogie (TP). D'après eux, cela confirme l'idée qu'il existe bien des liaisons entre ces dimensions (discipline et technologie) et qu'il ne s'agit pas de domaines distincts. La redondance de la dimension $\mathrm{T}$ dans cette corrélation peut faire l'objet d'une discussion. Une manière de contrôler la réelle corrélation entre TD et TP consisterait à procéder à des tests psychométriques pour vérifier dans quelle mesure le $\mathrm{T}$ associé à $\mathrm{D}$ peut être comparé au $\mathrm{T}$ associé à $\mathrm{P}$.

\section{Conclusion}

Le modèle du savoir technopédagogique disciplinaire que nous proposons intègre les différentes dimensions présentes dans les recherches antérieures (Shulman, 1986; Berthiaume, 2007; Mishra \& Koehler, 2006). Il s'agit de combiner les dimensions discipline (D), épistémologie personnelle (E), connaissances pédagogiques (P) et connaissances technologiques $(\mathrm{T})$ et de reconnaitre qu'il peut exister des relations plus ou moins fortes entre ces différentes composantes en fonction des profils des enseignants. Toutes ces dimensions sont présentes dans les profils des enseignants, mais elles n'ont pas la même importance.

Revue internationale de pédagogie de l'enseignement supérieur, 30(2) | 2014 


\subsection{Principaux résultats}

Sur la base d'un questionnaire composé de 28 items et de tests de corrélation entre les différentes dimensions, il en ressort que la dimension « connaissances pédagogiques » est corrélée de manière positive et significative avec les trois autres dimensions (connaissances technologiques, l'épistémologie personnelle et la discipline). L'étude des corrélations confirme également trois supposés :

1. Les nombreux travaux de Lebrun $(2006,2007 a, 2007 b, 2012)$ montraient déjà l'idée d'une relation entre pédagogies et technologies. Nous pouvons prouver ce lien dans notre recherche au travers des réponses d'enseignants de l'enseignement supérieur ;

2. La recherche de Berthiaume (2006) réintroduisait toute l'importance des références et des valeurs personnelles concernant la construction des savoirs dans la dynamique du savoir pédagogique disciplinaire (SPD). Nous montrons également que les enseignants associent cette dimension (E) aux connaissances pédagogiques, mais également à la discipline. Ceci valide le modèle SPD ;

3. Dans l'enseignement il est également supposé de longue date qu'il existe un lien très fort entre la discipline/contenu et les connaissances pédagogiques. Ceci rejoint les travaux de Lenze (1995) et de Shulman (1986). Nous trouvons bien une corrélation positive et fortement significative entre ces deux dimensions.

Par ailleurs, il apparaît clairement que la place des connaissances technologiques n'a de sens que s'il y a des connaissances pédagogiques. En effet, les connaissances technologiques seules ne sont pas corrélées avec la discipline et elles sont corrélées, mais pas de manière significative, avec l'épistémologie personnelle. Par contre, dès qu'elles sont associées à la pédagogie (TP), elles montrent une corrélation forte avec la relation technologie-épistémologie ( $\left.\mathrm{TE} r=0,773^{* *}\right)$, et cette dernière est, par ailleurs, fortement reliée à la relation technologie-discipline (TD $\left.r=694^{* *}\right)$. À ce niveau, nous avons discuté de la présence systématique de la dimension technologique dans ces trois relations. Une étude psychométrique des items permettrait de réellement considérer qu'il existe une corrélation valable. Dans les perspectives de recherches ultérieures, ces résultats seront évoqués comme des pistes potentielles pour expliquer les développements technopédagogiques des enseignants.

\subsection{Pistes en matière d'accompagnement pédagogique}

71 L'analyse en profil STPD semble apporter des éléments importants pour guider le conseiller pédagogique dans ses activités. Des chercheurs comme Jacquinot-Delaunay (2008) ou Rege Colet et Lenzo Marchese (2006) ont montré toute l'importance stratégique d'apporter un aspect disciplinaire aux formations aux technologies de l'information et de la communication (TIC) pour que celles-ci soient bien intégrées (Jacquinot-Delaunay, 2008) et parce qu'il existe des besoins spécifiques différents en fonction des facultés (Rege Colet \& Lenzo Marchese, 2006). La comparaison des usages des TIC (recherche documentaire en ligne, supports de cours, site web, forum, etc.) dans les différentes facultés de l'Université de Genève a, en effet, mis en évidence « des différences d'utilisation des technologies éducatives par faculté dont certaines sont très marquées » (p.14).

72 Autrement dit, pour l'accompagnement des enseignants universitaires, le conseiller pédagogique devrait pouvoir associer concepts pédagogiques, besoins disciplinaires, et 
également prendre en compte les conceptions des enseignants aux pratiques d'enseignement en ligne et à distance. Pour gérer toute cette complexité, Kanuka (2006) propose que les $\mathrm{CP}$, appelés dans son étude concepteurs pédagogiques, s'appuient sur un modèle comme le Pedagogical Content Knowledge (PCK) de Shulman pour former et accompagner les enseignants de l'université. Cela signifie que les conseillers et accompagnateurs pédagogiques ont besoin de développer non seulement des stratégies pédagogiques et les théories de l'apprentissage, mais également un répertoire de stratégies pédagogiques qui reflèteraient leur connaissance de la matière enseignée et de la culture de chaque discipline. Ceci parce que, selon Kanuka, l'expertise en technopédagogie ne doit pas être appliquée de la même manière dans toutes les disciplines.

73 Cette conception du métier est très interpellante, mais surtout très complexe à mettre en place dans la pratique. En partant de nos activités sur le terrain, nous avions l'impression de fonctionner de manière purement intuitive. C'est la raison pour laquelle, riche des différentes théories qui s'inspirent de Shulman (1986), nous avons réalisé ce modèle-outil.

74 Après avoir dressé le profil d'un enseignant avec le questionnaire et le radar, le conseiller pédagogique pourrait davantage orienter ses présentations d'outils ou de méthodes en référence avec le fonctionnement estimé de l'enseignant. En effet, le profil en radar réalisé à un moment donné pour un enseignant permet au conseiller pédagogique d'identifier les relations que l'enseignant a tendance à faire de manière plus prononcée (lien entre pédagogie et discipline, ou lien entre pédagogie et épistémologie, etc). Ainsi, il peut s'appuyer sur les relations fortes pour présenter certains outils technologiques en référence au mode de fonctionnement de l'enseignant. Plutôt que de fonctionner de manière intuitive dans l'accompagnement technopédagogique, cet outil permet de savoir si les choix des enseignants sont davantage orientés en fonction de leurs croyances épistémologiques, de la culture disciplinaire ou de ce qu'ils savent de la pédagogie.

\subsection{Perspectives et limites}

Le modèle-outil que nous proposons (le STPD) est une piste qui peut faire l'objet de nouvelles recherches. Les validations réalisées sont encourageantes, mais c'est réellement dans son utilisation que nous pourrons évaluer sa portée. Les items concernant la relation discipline-épistémologie pourraient, par exemple, faire l'objet d'une révision puisque l'alpha de Cronbach était plus faible (sous 0,7 ) pour cette relation. Nous les avons toutefois maintenus, car nous avons estimé qu'il était important de tester cette relation auprès de nos enseignantes. Par ailleurs, le test a été validé dans une Haute ÉCOLE qui propose des enseignements du niveau supérieur universitaire et non universitaire. Nous nous demandons si nous aurions obtenu les mêmes résultats auprès d'un public uniquement composé d'enseignants-chercheurs universitaires.

Cette étude montre également qu'il existe bien une compétence technopédagogique. Les connaissances pédagogiques et les connaissances technologiques montraient effectivement un lien dans le test de corrélation. Enfin, les applications réalisées ailleurs - des études de cas dans des disciplines différentes (Bachy, 2013) - nous encouragent à proposer cet outil à d'autres conseillers pédagogiques en vue de pouvoir 
davantage le tester et de pouvoir en discuter (ce qui n'a pas encore été possible jusque maintenant). Sur la base des retours des praticiens, des améliorations pourront être apportées. Pour accompagner la scénarisation et le travail avec les enseignants, il nous semble que nous possédons maintenant un outil qui nous permettrait de mieux comprendre les stratégies pédagogiques des enseignants. À partir de ces éléments, il nous est plus facile d'aborder des méthodes pédagogiques et de montrer des outils sur la plateforme e-learning.

\section{BIBLIOGRAPHIE}

Amade-Escot, C. (2011, octobre). Le comparatisme en didactique. Présentation dans le cadre de l'école doctorale des didactiques, Liège, Belgique.

Archambault, L. \& Crippen, K. (2009). Examining TPACK among K-12 online distance educators in the United States Contemporary Issues. Technology and Teacher Education, 9(1), 71-88.

Bachy, S. (2013). L'enseignement en ligne à l'université et les spécificités disciplinaires (Thèse de doctorat inédit). Université catholique de Louvain.

Becher, T. (1981). Toward a definition of disciplinary cultures. Studies in Higher Education, 6(2), 109-122.

Becher, T. (1993). Academic Tribes and Territories. Buckingham : Society of Research into Higher Education and Open university Press.

Becher, T. \& Trowler, P. R. (2001). Academic tribes and territories: Intellectual enquiry and the cultures of disciplines (2nd ed.) Buckingham : Society of Research into Higher Education and Open university Press.

Berthiaume, D. (2006). A description of discipline-specific pedagogical knowledge (DPK)encountered in the discourse of four university professors from four different disciplinary areas (Thèse de doctorat non publiée). University de Mc Gill, Montréal.

Berthiaume, D. (2007, mai). Une description empirique du savoir pédagogique disciplinaire des professeurs d'université. Dans Actes du colloque de l'AIPU : regards sur l'innovation la collaboration et la valorisation (p.179-181). Montréal : Canada.

Biglan, A. (1973). The characteristics of subject matter in different academic areas. Journal of Applied Psychology, 57(3), 195-203.

Bourdieu, P. (1984). Homo Academicus. Paris : Editions de Minuit.

Bourgeois, E. (1990). University politics: adult Education in a Belgian University (Thèse non publiée). University of Chicago : Chicago.

Cochran, K.F., King, R.A. \& De Ruiter, J.A. (1993). Pedagogical content knowledge: an integrative model for teacher preparation. Journal of teacher Education, 44(4), 263-272.

Collès, L. \& Bachy, S. (2011). Le master FLE-Francophonie de l'université catholique de Louvain. Dans C. Eid (dir.), Didactique et TICE IV des 13 et 14 mai 2010, Les programmes de formation universitaire (p. 209-230). Eid : Beyrouth. 
Czaja, R. \& Blair, J. (2005). Designing surveys: A guide to decisions and procedures (2nd ed.). Thousand Oaks, CA: Sage Publications.

Donald, J. (2002). Learning to thinking. Disciplinary Differences. San Fransisco, CA : Jossey-Bass.

Gagné, R. (1985). The conditions of learning. New York : Holt Rinehart and Winston.

Gess-Newsome, J. \& Lederman, N.G. (1999). Examining pedagogical content knowledge: The Construct and its implications for science education. Dordrecht, NL : Kluwer.

Graham, C. R., Borup, J. \& Smith, N. B. (2012). Using TPACK as a framework to understand teacher candidates technology integration decisions. Journal of Computer Assisted Learning, 28, 530-546.

Guyot, JL. \& Bonami, M. (2000). Mode de structuration du travail professoral et logiques disciplinaires à l'université. Cahier du GIRSEF,9, 1-65.

Harris, J., Grandgenett, N. \& Hofer, M. (2010). Testing a TPACK-based technology Integration Assessment rubric. Dans D. Gibson et B. Dodge (dir.), Proceedings of Society for Information Technology \& Teacher Education International Conference 2010 (p.3833-3840), Chesapeake, VA.

Hativa, N. \& Marincovich, M. (Eds.) (1995). Disciplinary differences in teaching andlearning: Implications for practice. San Francisco, CA: Jossey-Bass.

Hiebert, J., Gallimore, R. \& Stigler, J. W. (2002). A Knowledge base for the teachingprofession: what would it look like and how can we get one? Educational Researcher, 31(5), 3-15.

Hofer, B.K. (2004). Epistemological understanding as a metacognitive process : Thinking aloud during online searching. Educational Psychologist, 29(1), p.43-55.

Hofer, B.K. \& Pintrich, P. R. (1997). The Development of epistemological theories: Beliefs about knowledge and knowing and their relation to learning. Review of Educational Research, 67(1), 88-140.

Hofer, B.K. \& Pintrich, P. R. (2002). Personal epistemology: The psychology of beliefs about knowledge and knowing. Mahwah, NJ: Lawrence Earlbaum Associates.

Hofer, M. \& Harris, J. (2010) Differentiating TPACK development: using learning activity types with inservice and preservice teachers. Dans D. Gibson et B. Dodge (dir.), Proceedings of Society for Information Technology \& Teacher Education International Conference 2010 (p. 3857-3864), Chesapeake, VA.

Jacquinot-Delaunay, G. (2008). L'intégration des TICE dans l'institution universitaire : de l'infiltration à l'innovation? Dans G. Delaunay et E. Fichez (dir.), L'université et les TIC. Chronique d'une innovation annoncée (p.179-222). Bruxelles : De Boeck.

Kanuka, H. (2006). Instructional design and e-learning : a discussion of pedagogical content knowledge as a missing construct. The e-Journal of Instructional Science and Technology,9(2). Repéré à http://www.ascilite.org.au/ajet/e-jist/docs/vol9_no2/papers/full_papers/kanuka.htm.

Khun, T.S. (1962). The structure of scientific Revolutions. Chicago : Chicago University Press. Kolb, D.-A. (1981). Learning styles and disciplinary differences. Dans A. W. Chickering (ed.) The Modern American College (p. 232-255). San Francisco, CA: Jossey-Bass.

Lebrun, M. (2002). Des technologies pour enseigner et apprendre (2 ${ }^{\text {ème }}$ ed.). Bruxelles: De Boeck. Lebrun, M. (2006). E-Learning pour enseigner et pour apprendre. Allier pédagogie et technologie. Louvain-la-Neuve : Ed. Bruylant-academia. 
Lebrun, M. (2007a). Quality towards an expected harmony: pedagogy and technology speaking together about innovation. AACE Journal, 15(2), 115-130.

Lebrun, M. (2007b). Théories et méthodes pédagogiques pour enseigner et apprendre. Quelle place pour les TIC dans l'éducation? Bruxelles : De Boeck.

Lebrun, M. (2012). Classes inversées, Flipped Classrooms ...Ca flippe quoi au juste ? Repéré à http:// lebrunremy.be/WordPress/

Lenze, L. F. (1995). Discipline-specific pedagogical knowledge in Linguistics and Spanish. Dans N. Hativa \& M. Marincovich (dir.), Disciplinary differences in teaching and learning: Implications for practice (p. 65-70). San Francisco, CA: Jossey-Bass.

Lessard, C., Altet, M., Paquay, L. \& Perrenoud, P. (2004). Entre sens commun et sciences humaines. Quels savoirs pour enseigner? Bruxelles : De Boeck.

Magnusson, S., Krajcik, J. \& Borko, H. (1999). Nature, sources, and development of pedagogical content knowledge for science teaching. Dans J. Gess-Newsome \& N.G. Lederman (dir.), Examining pedagogical content knowledge (p.95-132). Dordrecht, NL: Kluwer.

Milken family foundation and the international society for Technology (1999). Will new teachers be prepared to teach in a digital age?Repéré à http://eric.ed.gov/?id=ED428072

Mishra, P. \& Koehler, M. J. (2006). Technological pedagogical content knowledge: A framework for integrating technology in teacher knowledge. Teachers College Record,108(6), 1017-1054.

Munby, H., Russell, T. \& Martin, A. K. (2001). Teachers' knowledge and how it develops. Dans V. Richardson (dir.), Handbook of Research on Teaching (4 ed. p.877-904). Washington, DC: American Educational Research Association.

Neumann, R. (2001). Disciplinary differences and university teaching. Studies in Higher Education, 26(2), 135-146.

Niess, M.L. (2005). Preparing teachers to teach science and mathematics with technology: Developing a technology pedagogical content knowledge. Teaching and Teacher Education,21, 509-523.

Puentedura, R. (2006). Transformation, Technology and Education [diaporama]. Repéré à http:// hippasus.com/resources/tte/.

Rege Colet, N. \& Lenzo Marchese, G. (2006). Peut-on parler de spécificités disciplinaires dans l'utilisation de TIC ? Études des pratiques facultaires à l'Université. Actes du XXIIIème congrès AIPU 2006. Repéré à www.unige.ch/formev/publications/technologies/texte-regecolet-lenzo.pdf. Sensevy, G. (2009). Didactique et sciences de l'éducation : une reconfiguration ? Dans A. Vergnioux (dir.), 40 ans des sciences de l'éducation (p. 49-58). Caen : Presses universitaires de Caen. Schmidt, D. A., Baran, E., Thompson, A. D., Mishra, P., Koehler, M. \& Shin, T. S. (2009). Technological pedagogical content knowledge (TPACK): The development and validation of an assessment instrument for preservice teachers. Journal of Research on Technology in Education, 42(2), 123-149.

Schwab, J.J. (1964). Structure of the disciplines : meanings and significances. Dans G.W. Ford \& L. Pugno (dir.), The structure of knowledge and the curriculum(p.6-30). Chicago : Rand Mc Nally.

Segall, A. (2004). Revisiting pedagogical content knowledge: the pedagogy of content/the content of pedagogy. Teaching and Teacher Education,20, 489-504. 
Shulman, L. (1986). Those who understand: Knowledge growth in teaching. Educational researcher, 15(2), 4-14.

Shulman, L.S. (1987). Knowledge and teaching: foundations of the new reform. Harvard Educational review, 57, 1-22.

Shulman, L.S. (1998). Theory, practice and education of professionals. The Elementary School Journal, 98(5), 511-526.

Shulman, L.S. (2004). How and what teachers learn : a shifting perspective. Journal of Curriculum studies, 36(2), 257-271.

Shulman, L.S. (2007). Ceux qui comprennent :Le développement de la connaissance dans l'enseignement. Education et Didactique, 1(1), 97-114.

Van Driel, J.H., Verloop, N. \& Devos, W. (1998). Developing science teachers' pedagogical content knowledge. Journal of research in science Teaching, 35(6), 673-695.

Vause, A. (2009). Les croyances et connaissances des enseignants à propos de l'acte d'enseigner. Les Cahiers de Recherche en Éducation et Formation, 66.

\section{RÉSUMÉS}

Le modèle du savoir technopédagogique disciplinaire que nous proposons intègre différentes dimensions qui peuvent soutenir notre compréhension des pratiques des enseignants. Il s'agit de combiner les dimensions: discipline (D), épistémologie personnelle (E), connaissances pédagogiques $(\mathrm{P})$ et connaissances technologiques $(\mathrm{T})$ et de reconnaître qu'il peut exister des relations plus ou moins fortes entre ces différentes composantes en fonction des profils des enseignants. Toutes ces dimensions sont présentes dans les profils des enseignants, mais elles n'ont pas la même importance. Pour déterminer les différents profils des enseignants, nous avons élaboré un questionnaire pour tester chaque dimension et chaque relation entre ces dimensions. Le questionnaire a fait l'objet d'une première validation pour vérifier si les items étaient compréhensibles et si les profils qui en sortaient étaient fidèles aux caractéristiques que nous connaissions chez les enseignants-témoins. Une deuxième validation a été réalisée sur la base de réponses d'enseignants de l'enseignement supérieur universitaire et non universitaire. L'objectif était d'analyser la cohérence entre les regroupements des items et les corrélations entre les différentes dimensions du modèle. Il en ressort que la dimension " connaissances pédagogiques » est corrélée de manière positive et significative avec les trois autres dimensions (connaissances technologiques, l'épistémologie personnelle et la discipline). L'utilisation du modèle-outil nous a permis d'affiner notre travail d'accompagnateur pédagogique dans le cadre de la mise en place de cours universitaires supportés par les technologies.

The new Technopedagogical Disciplinary Knowledge model that we propose integrates different dimensions: discipline (D), personal epistemology (E), pedagogical knowledge (P) and technology knowledge $(\mathrm{T})$. We acknowledge the existence of relationships between the components of the model that can be more or less strong according to the profiles of teachers. All these dimensions are present in teachers' profiles, but they do not have the same importance. In order to establish the different profiles of teachers we designed a questionnaire to test the component and the relationships. The questionnaire was submitted a first time in order to check if the items were understandable and if the profiles that emerged were reliable based on what we knew of and about the teachers. A second test was carried out based on comparing the answers of university and non-university teachers. The purpose was to test the consistency between groups of items 
and the correlations between the different dimensions of the model. It appears that the "pedagogical knowledge" dimension is significantly correlated with the other three dimensions (technological knowledge, personal epistemology and discipline). This consolidated framework has help build up education development coaching in teaching practices that imply technology.

\section{INDEX}

Mots-clés : accompagnement pédagogique, e-learning, modèle-outil, savoir pédagogique disciplinaire

\section{AUTEUR}

\section{SYLVIANE BACHY}

Institut de recherche Cripedis et Institut de pédagogie universitaire et des multimédias Université catholique de Louvain, Place Montesquieu 1, B-1348 Louvain-la-Neuve (Belgique) Sylviane.bachy@uclouvain.be 